\title{
Graph Energy and Hadamard's Inequality
}

\author{
Amir Hossein Ghodrati \\ Department of Mathematics, Faculty of Science, \\ Shahid Rajaee Teacher Training University, Tehran, Iran \\ ghodrati@sru.ac.ir
}

(Received November 12, 2021)

\begin{abstract}
We use Hadamard's determinantal inequality and its generalization to prove some upper bounds on the energy of a graph in terms of degrees, average 2-degrees and number of common neighbors of its vertices. Also, we prove an inequality relating the energy of a graph and one arbitrary subgraph of it.
\end{abstract}

\section{Introduction}

To a graph $G$ (which is always assumed to be finite, simple and undirected) with the vertex set $\{1, \ldots, n\}$, we can assign a symmetric $(0,1)$ matrix $A$, which is called the adjacency matrix of $G$, whose $(i, j)$ entry is one if and only if $i$ and $j$ are adjacent. The energy of $G$, which is introduced by Gutman in [7] and is denoted by $\mathcal{E}(G)$, is the sum of absolute values of the eigenvalues of $A$.

As is mentioned by Nikiforov in [15], the energy of a graph is the same as the trace norm of its adjacency matrix. The trace norm of a general complex square matrix $A$ is defined as the sum of its singular values. In this paper, we use the term energy of $A$ to refer to the trace norm of $A$ and denote this quantity by $\mathcal{E}(A)$.

There are many lower and upper bounds for the energy of a graph, see for example Chapter 5 of [10]. The purpose of this paper is to investigate the application of Hadamard's inequality and its generalizations for finding upper bounds for the energy of graphs and matrices in general. The Hadamard's inequality can be stated in two equivalent forms, which are presented in the following theorem. Note that $M_{n}(\mathbb{C})$ denotes the set of all $n \times n$ 
complex matrices, and the Euclidean norm of a complex vector $\left(c_{1}, \ldots, c_{n}\right)$ is defined as $\sqrt{\sum_{i=1}^{n}\left|c_{i}\right|^{2}}$.

Theorem 1. (Hadamard's inequality)

(i) Let $A=\left[a_{i j}\right] \in M_{n}(\mathbb{C})$ be positive definite. Then

$$
\operatorname{det}(A) \leq a_{11} \cdots a_{n n} .
$$

(ii) Let $A \in M_{n}(C)$ and $r_{i}$ be the Euclidean norm of the $i$-th row of $A$. Then

$$
|\operatorname{det}(A)| \leq r_{1} \cdots r_{n}
$$

For more information about this fundamental inequality, its proof and some of its generalizations, see [11, Section 7.8].

In Section 2 of this paper, we use an intermediate Lemma appeared in [16] for the proof of the Coulson integral formula to express the energy of a matrix as an integral involving a determinant, and using that expression and applying the Hadamard's inequality we find some upper bounds for the energy of a matrix and a graph. In Section 3, we use a generalization of the Hadamard inequality, namely the Fischer's inequality, to prove an upper bound for the energy of a graph which involves the number of common neighbors of some of the vertices. Finally in Section 3, we use an inequality about the singular values of the Hadamard product of two matrices to find a relation between the energy of a graph and the energy of one of its subgraphs.

\section{Upper bounds on the energy using Hadamard's in- equality}

One of the fundamental formulas for computing the energy of a graph is the Coulson integral formula [5]. This formula expresses the the energy of a graph as an integral involving the characteristic polynomial of that graph. The following Lemma is used in [16] to prove the Coulson integral formula.

Lemma 2. [16, Lemma 5.3.2] For every real number a,

$$
|a|=\frac{1}{\pi} \int_{0}^{\infty} \frac{1}{x^{2}} \ln \left(1+x^{2} a^{2}\right) d x .
$$

This lemma enables us to prove the following theorem for the energy of a general complex square matrix. Note that similar formulas are reported in [8] for the adjacency matrices of general graphs and in [6] for acyclic graphs. 
Theorem 3. For $A \in M_{n}(\mathbb{C})$,

$$
\mathcal{E}(A)=\frac{1}{\pi} \int_{0}^{\infty} \frac{1}{x^{2}} \ln \operatorname{det}\left(I+x^{2} A A^{*}\right) d x
$$

Proof. Let $\sigma_{1}, \ldots, \sigma_{k}$ be the nonzero singular values of $A$. So $\sigma_{1}^{2}, \ldots, \sigma_{k}^{2}$ are nonzero eigenvalues of $A A^{*}$ and for every real number $x$,

$$
\operatorname{det}\left(I+x^{2} A A^{*}\right)=\prod_{j=1}^{k}\left(1+x^{2} \sigma_{j}^{2}\right) .
$$

Now by Lemma $2, \sigma_{j}=\frac{1}{\pi} \int_{0}^{\infty} \frac{1}{x^{2}} \ln \left(1+\sigma_{j}^{2} x^{2}\right) d x$, for every $j \in\{1, \ldots, k\}$, which implies that

$$
\begin{aligned}
\mathcal{E}(A)=\sum_{j=1}^{k} \sigma_{j} & =\frac{1}{\pi} \int_{0}^{\infty} \frac{1}{x^{2}} \ln \prod_{j=1}^{k}\left(1+x^{2} \sigma_{j}^{2}\right) d x \\
& =\frac{1}{\pi} \int_{0}^{\infty} \frac{1}{x^{2}} \ln \operatorname{det}\left(I+x^{2} A A^{*}\right) d x
\end{aligned}
$$

as desired.

The integrand in Theorem 3 contains the determinant of a positive definite matrix, and this determinant can be bounded using the Hadamard's inequality. This leads us to the next theorem.

Theorem 4. Let $A \in M_{n}(\mathbb{C})$ and denote the Euclidean norm of the $i$-th row of $A$ by $r_{i}$. Then

$$
\mathcal{E}(A) \leq \sum_{i=1}^{n} r_{i}
$$

Proof. For every real number $x$, the diagonal entries of the positive definite matrix $I+$ $x^{2} A A^{*}$ are $1+x^{2} r_{1}^{2}, \ldots, 1+x^{2} r_{n}^{2}$. So by the Hadamard's inequality, $\operatorname{det}\left(I+x^{2} A A^{*}\right) \leq$ $\prod_{i=1}^{n}\left(1+x^{2} r_{i}^{2}\right)$. Now, Theorem 3 and Lemma 2 give

$$
\mathcal{E}(A)=\frac{1}{\pi} \int_{0}^{\infty} \frac{1}{x^{2}} \ln \operatorname{det}\left(I+x^{2} A A^{*}\right) d x \leq \frac{1}{\pi} \int_{0}^{\infty} \frac{1}{x^{2}} \ln \prod_{i=1}^{n}\left(1+x^{2} r_{i}^{2}\right) d x=\sum_{i=1}^{n} r_{i},
$$

which completes the proof.

For a Hermitian matrix $A, \mathcal{E}(A)$ is the sum of absolute values of the eigenvalues of $A$. In some cases, it is desirable to consider a matrix similar to $A$ and use the sum of Euclidean norms of the rows of that matrix to find an upper bound for the energy. But, although the eigenvalues are invariant under a similarity transformation, singular values are not, and so the energy may change by such a transformation. In this cases the following corollary could be helpful. 
Corollary 5. Let $A \in M_{n}(\mathbb{C})$ has the eigenvalues $\lambda_{1}, \ldots, \lambda_{n}$. If the Euclidean norm of the $i$-th row of $A$ is $r_{i}$, then

$$
\sum_{i=1}^{n}\left|\lambda_{i}\right| \leq \sum_{i=1}^{n} r_{i}
$$

In particular, if $A$ is Hermitian, $P$ is an invertible $n \times n$ matrix and the Euclidean norm of the $i$-th row of $P^{-1} A P$ is $r_{i}$, then

$$
\mathcal{E}(A) \leq \sum_{i=1}^{n} r_{i} .
$$

Proof. Assume that $\lambda_{1} \geq \cdots \geq \lambda_{k}$ be the nonzero eigenvalues of $A$. Also, let $\sigma_{1} \geq$ $\cdots \geq \sigma_{k}$ be the positive singular values of $A$. By a theorem attributed to Weyl in $[2$, p.43], $\left(\sigma_{1}, \ldots, \sigma_{k}\right)$ log-majorizes $\left(\left|\lambda_{1}\right|, \ldots,\left|\lambda_{k}\right|\right)$. But by [13, p. 168, A.2.b], weak logmajorization implies weak majorization. So $\left(\sigma_{1}, \ldots, \sigma_{k}\right)$ weakly majorizes $\left(\left|\lambda_{1}\right|, \ldots,\left|\lambda_{k}\right|\right)$. In particular,

$$
\sum_{i=1}^{k}\left|\lambda_{i}\right| \leq \sum_{i=1}^{k} \sigma_{k}=\mathcal{E}(A) .
$$

This inequality together with Theorem 4 give the first part.

For the second part, note that the energy of the Hermitian matrix $A$ is the sum of absolute values of its eigenvalues, and the eigenvalues of $A$ and $P^{-1} A P$ are the same. So using the first part for $P^{-1} A P$ gives the result.

In [1] the energy of a complex square matrix is defined to be the sum of absolute values of its eigenvalues. Theorem 5 shows that the sum of Euclidean norms of the rows of a matrix is an upper bound for this type of energy too.

The following upper bound for the energy of a graph is obtained in [3] and [4] using the concept of the energy of a vertex. Here, we present a new proof based on Theorem 4.

Theorem 6. If $G$ is a graph with degree sequence $d_{1}, \ldots, d_{n}$, then

$$
\mathcal{E}(G) \leq \sum_{i=1}^{n} \sqrt{d_{i}} .
$$

Proof. The Euclidean norm of the $i$-th row of the adjacency matrix of a graph is the square root of the degree of its $i$-th vertex. So Theorem 4 gives the result.

One of the simplest upper bounds for the energy of a graph is the McClelland's bound [14], which states that for every graph $G$ with $n$ vertices and $m$ edges, $\mathcal{E}(G) \leq \sqrt{2 n m}$. Since Cauchy-Schwarz inequality implies that $\sum_{i=1}^{n} \sqrt{d_{i}} \leq \sqrt{n \sum_{i=1}^{n} d_{i}}=\sqrt{2 n m}$, the 
bound in Theorem 6 is better than McClelland's bound. Another simple upper bound for the energy of a graph in terms of the number of vertices and the number of edges is stated in the following theorem.

Theorem 7. [9] If $2 m \geq n$ and $G$ is a graph of order $n$ and size $m$, then

$$
\mathcal{E}(G) \leq \frac{2 m}{n}+\sqrt{(n-1)\left[2 m-\left(\frac{2 m}{n}\right)^{2}\right]}
$$

Computation shows that among 11716573 connected graphs with at most 10 vertices, there are only 74518 graphs for which the upper bound in Theorem 6 is better than the upper bound given in Theorem 7, so Theorem 6 is rather weak. In the next section we try to use a generalization of the Hadamard's inequality to find a better upper bound. To see another upper bound for the energy of a graph which depends on the degree sequence, see $[19]$.

Other quantities that have been used to find upper bounds for the energy of a graph are that of the 2-degrees and the average 2-degrees of the vertices (see [17]). The 2-degree of a vertex is the sum of the degrees of its neighbors, and the average 2-degree of that vertex is the arithmetic mean of the degrees of its neighbors (provided that the vertex is not isolated). In the following theorem, we prove an upper bound for the energy in terms of the average 2-degrees of the vertices. The expression is quiet similar to that of Theorem 6.

Theorem 8. Let $G$ be a graph with the vertex set $\{1, \ldots, n\}$ and without isolated vertices, and let $m_{i}$ be the average 2-degree of $i$. Then,

$$
\mathcal{E}(G) \leq \sum_{i=1}^{n} \sqrt{m_{i}}
$$

Proof. Let $d_{i}$ be the degree of $i$ and let $P$ be a diagonal matrix with the diagonal entries $\sqrt{d_{1}}, \ldots, \sqrt{d_{n}}$. If $A=\left[a_{i j}\right]$ is the adjacency matrix of $A$, then $P^{-1} A P=\left[a_{i j} \frac{\sqrt{d_{j}}}{\sqrt{d_{i}}}\right]$, and the square of the Euclidean norm of the $i$-th row of this matrix is

$$
\sum_{j=1}^{n} a_{i j}^{2} \frac{d_{j}}{d_{i}}=\frac{1}{d_{i}} \sum_{j=1}^{n} a_{i j} d_{j}=m_{i}
$$

So by Corollary $5, \mathcal{E}(G) \leq \sum_{i=1}^{n} \sqrt{m_{i}}$. 


\section{$3 \quad$ Energy upper bound using Fischer's inequality}

In this section we use the Fischer's inequality, which is a generalization of the Hadamard's inequality, to find another upper bound for the energy of a graph. For a proof of the Fischer's inequality, see [11, Theorem 7.8.5] and the exercise following it.

Theorem 9. Let $M$ be a positive definite matrix in the following block form:

$$
M=\left(\begin{array}{ccc}
M_{11} & \cdots & M_{1 k} \\
\vdots & \ddots & \vdots \\
M_{k 1} & \cdots & M_{k k}
\end{array}\right)
$$

and assume that $M_{11}, \ldots, M_{k k}$ are square matrices. Then, $\operatorname{det}(M) \leq \operatorname{det}\left(M_{11}\right) \cdots \leq$ $\operatorname{det}\left(M_{k k}\right)$.

The following lemma is a generalization of Lemma 2 .

Lemma 10. Let $f$ be a polynomial of degree $n$ with negative real roots $-c_{1}, \ldots,-c_{n}$, and suppose that $f(0)=1$. Then,

$$
\frac{1}{\pi} \int_{0}^{\infty} \frac{1}{x^{2}} \ln f\left(x^{2}\right) d x=\sum_{i=1}^{n} \frac{1}{\sqrt{c_{i}}} .
$$

Proof. Let $a$ be the leading coefficient of $f$. Then,

$$
\begin{aligned}
f(x)=a\left(x+c_{1}\right) \cdots\left(x+c_{n}\right) & =a c_{1} \cdots c_{n}\left(1+\frac{x}{c_{1}}\right) \cdots\left(1+\frac{x}{c_{n}}\right) \\
& =\left(1+\frac{x}{c_{1}}\right) \cdots\left(1+\frac{x}{c_{n}}\right),
\end{aligned}
$$

since $a c_{1} \ldots c_{n}=f(0)=1$. Now, using Lemma 2 we get

$$
\begin{aligned}
\frac{1}{\pi} \int_{0}^{\infty} \frac{1}{x^{2}} \ln f\left(x^{2}\right) d x & =\frac{1}{\pi} \int_{0}^{\infty} \frac{1}{x^{2}} \ln \prod_{i=1}^{n}\left(1+\frac{x^{2}}{c_{i}}\right) d x \\
& =\sum_{i=1}^{n} \frac{1}{\pi} \int_{0}^{\infty} \frac{1}{x^{2}} \ln \left(1+\frac{x^{2}}{c_{i}}\right) d x=\sum_{i=1}^{n} \frac{1}{\sqrt{c_{i}}},
\end{aligned}
$$

and this completes the proof.

A matching over $\{1, \ldots, n\}$ is a set of disjoint two-element subsets of $\{1, \ldots, n\}$. An element of $\{1, \ldots, n\}$ is said to be covered by $M$ if it belongs to one of the sets in $M$.

Theorem 11. Let $G$ be a graph with the vertex set $\{1, \ldots, n\}, M$ be a matching over $\{1, \ldots, n\}$, and $N$ be the vertices not covered by $M$. Also, suppose that $d_{i}$ is the degree of $i$ and $d_{i j}$ is the number of common neighbors of $i$ and $j$. Then

$$
\mathcal{E}(G) \leq \sum_{i \in N} \sqrt{d_{i}}+\sum_{\{i, j\} \in M} \sqrt{d_{i}+d_{i}+2 \sqrt{d_{i} d_{j}-d_{i j}^{2}}}
$$


Proof. Without the loss of generality we can assume that $M=\{\{1,2\},\{3,4\}, \ldots,\{2 r-$ $1,2 r\}$. Let $A$ be the adjacency matrix of $G$. The matrix $A A^{*}=A^{2}$ can be written in the following block form:

$$
A^{2}=\left(\begin{array}{cccccc}
B_{1} & & & & & \\
& \ddots & & & * & \\
& & B_{r} & & & \\
& & & d_{2 r+1} & & \\
& * & & & \ddots & \\
& & & & & d_{n}
\end{array}\right)
$$

where $B_{i}=\left(\begin{array}{cc}d_{2 i-1} & d_{2 i-1,2 i} \\ d_{2 i-1,2 i} & d_{2 i}\end{array}\right)$. So by Theorem 3 and Fischer's inequality,

$$
\begin{aligned}
\mathcal{E}(G) & =\frac{1}{\pi} \int_{0}^{\infty} \frac{1}{x^{2}} \ln \operatorname{det}\left(I+x^{2} A^{2}\right) d x \leq \frac{1}{\pi} \int_{0}^{\infty} \frac{1}{x^{2}} \ln \left[\prod_{i=1}^{r} \operatorname{det}\left(I+x^{2} B_{i}\right) \prod_{i=2 r+1}^{n}\left(1+d_{i} x^{2}\right)\right] d x \\
& =\sum_{i=1}^{r} \frac{1}{\pi} \int_{0}^{\infty} \frac{1}{x^{2}} \ln \operatorname{det}\left(I+x^{2} B_{i}\right) d x+\sum_{i=2 r+1}^{n} \frac{1}{\pi} \int_{0}^{\infty} \frac{1}{x^{2}} \ln \left(1+d_{i} x^{2}\right) d x .
\end{aligned}
$$

By Lemma $2, \frac{1}{\pi} \int_{0}^{\infty} \frac{1}{x^{2}} \ln \left(1+d_{i} x^{2}\right) d x=\sqrt{d_{i}}$. Also, let

$$
f_{i}(x)=\operatorname{det}\left(I+x B_{i}\right)=\left(1+d_{s} x\right)\left(1+d_{t} x\right)-d_{s t}^{2} x^{2}
$$

where for simplifying the formulas we let $s=2 i-1$ and $t=2 i$. The reciprocals of the roots of $f_{i}$ are $-\frac{1}{2}\left(d_{s}+d_{t} \pm \sqrt{\left(d_{s}-d_{t}\right)^{2}+4 d_{s t}^{2}}\right)$, so be Lemma 10 ,

$$
\begin{aligned}
\frac{1}{\pi} \int_{0}^{\infty} \frac{1}{x^{2}} \ln f_{i}\left(x^{2}\right) d x & =\sqrt{\frac{d_{s}+d_{t}+\sqrt{\left(d_{s}-d_{t}\right)^{2}+4 d_{s t}^{2}}}{2}}+\sqrt{\frac{d_{s}+d_{t}-\sqrt{\left(d_{s}-d_{t}\right)^{2}+4 d_{s t}^{2}}}{2}} \\
& =\sqrt{d_{s}+d_{t}+2 \sqrt{d_{s} d_{t}-d_{s t}^{2}}}
\end{aligned}
$$

Therefore,

$$
\begin{aligned}
\mathcal{E}(G) & \leq \sum_{i=1}^{r} \sqrt{d_{2 i-1}+d_{2 i}+2 \sqrt{d_{2 i-1} d_{2 i}-d_{2 i-1,2 i}^{2}}}+\sum_{i=2 r+1}^{n} \sqrt{d_{i}} \\
& =\sum_{\{i, j\} \in M} \sqrt{d_{i}+d_{i}+2 \sqrt{d_{i} d_{j}-d_{i j}^{2}}}+\sum_{i \in N} \sqrt{d_{i}},
\end{aligned}
$$

as desired.

Note that

$$
\sqrt{d_{i}+d_{i}+2 \sqrt{d_{i} d_{j}-d_{i j}^{2}}} \leq \sqrt{d_{i}}+\sqrt{d_{j}}
$$


and the equality holds if and only if $d_{i j}=0$. So, Theorem 11 generally gives a better bound than Theorem 6. Also, the optimal result of Theorem 11 is obtained when $M$ is as large as possible. So when $n$ is even, one should choose a matching that covers all of $\{1, \ldots, n\}$, and when $n$ is odd, a matching that covers $n-1$ vertices should be chosen.

\section{Energy of a subgraph}

There is another way of looking at the Hadamard's inequality using the notion of Hadamard product. For $A=\left[a_{i j}\right], B=\left[b_{i j}\right] \in M_{n}(\mathbb{C})$, the Hadamard product (or Schur product) of $A$ and $B$ is defined as $A \circ B=\left[a_{i j} b_{i j}\right]$. Now, the Hadamard's inequality can be restated as follows: for every positive definite matrix $A, \operatorname{det}(A) \leq \operatorname{det}(A \circ I)$. There are many results concerning the eigenvalues and singular values of the Hadamard product. In this section, we use such a theorem to find an upper bound for the energy of a subgraph. Note that for $A \in M_{n}(\mathbb{C}), \sigma_{1}(A) \geq \cdots \geq \sigma_{n}(A)$ denote the singular values of $A$ in descending order. Also, let $r_{1}(A) \geq \cdots \geq r_{n}(A)$ be the Euclidean norms of the rows of $A$ in descending order.

Theorem 12. [12, Theorem 5.5.20] For any $n \times n$ complex matrices $A, B$,

$$
\sum_{i=1}^{n} \sigma_{i}(A \circ B) \leq \sum_{i=1}^{n} r_{i}(B) \sigma_{i}(A) .
$$

A generalization of this theorem is proved in in [18].

The energy of an induced subgraph of a graph $G$ is less than or equal to the energy of $G$ [10, Theorem 4.19], but similar inequality does not hold for an arbitrary subgraph (for example, see [16, p.199]). Nevertheless, we can find an inequality relating the energy of a graph and the energy of an arbitrary subgraph of it.

Theorem 13. Let $H$ be a subraph of $G$ with maximum degree $\Delta(H)$. Then

$$
\mathcal{E}(H) \leq \mathcal{E}(G) \sqrt{\Delta(H)}
$$

Proof. Since there is a spanning subgraph of $G$ which contains $H$ as an induced subgraph, and the energy of an induced subgraph is less than or equal to the the energy of its supergraph, we can assume that $H$ is a spanning subgraph of $G$. Let $A, B$ be the adjacency matrices of $G$ and $H$, respectively, with the same ordering of the vertices. It is clear that $A \circ B=B$, and the Euclidean norm of the $i$-th row of $B$ is the square root of the $i$-th vertex 
of $H$ which is at most $\sqrt{\Delta(H)}$. So, by Theorem $12, \sum_{i=1}^{n} \sigma_{i}(B) \leq \sum_{i=1}^{n} \Delta(H) \sigma_{i}(A)$, or $\mathcal{E}(H) \leq \sqrt{\Delta(H)} \mathcal{E}(G)$, as desired.

\section{References}

[1] S. Akbari, E. Ghorbani, M. R. Oboudi, Edge addition, singular values, and energy of graphs, Lin. Algebra Appl. 430 (2009) 2192-2199.

[2] T. Ando, Majorizations and inequalities in matrix theory, Lin. Algebra Appl. 199 (1994) $17-67$.

[3] O. Arizmendi, O. Juarez-Romero, On bounds for the energy of graphs and digraphs, in: F. Galaz-García, J. C. Pardo Millán, P. Solórzano (Eds.), Contributions of Mexican Mathematicians Abroad in Pure and Applied Mathematics, Am. Math. Soc., Providence, 2018, pp. 1-19.

[4] O. Arizmendi, J. F. Hidalgo, O. Juarez-Romero, Energy of a vertex, Lin. Algebra Appl. 557 (2018) 464-495.

[5] C. A. Coulson, On the calculation of the energy in unsaturated hydrocarbon molecules, Proc. Cambridge Phil. Soc. 36 (1986) 201-203.

[6] I. Gutman, Acyclic systems with extremal Hückel $\pi$-electron energy, Theor. Chim. Acta 45 (1977) 79-87.

[7] I. Gutman, The energy of a graph, Ber. Math.-Stat. Sekt. Forschungszent. Graz. 103 (1978) 1-22.

[8] I. Gutman, N. Trinajstić, Graph theory and molecular orbitals. XV. The Hückel rule, J. Chem. Phys. 64 (1976) 4921-4925.

[9] J. H. Koolen, V. Moulton, Maximal energy graphs, Adv. Appl. Math. 26 (2001) 47-52.

[10] X. Li, Y. Shi, I. Gutman, Graph Energy, Springer, New York, 2012.

[11] R. A. Horn, C. R. Johnson, Matrix Analysis, Cambridge Univ. Press, Cambridge, 2013.

[12] R. A. Horn, C. R. Johnson, Topics in Matrix Analysis, Cambridge Univ. Press, Cambridge, 1991.

[13] A. W. Marshall, I. Olkin, B. C. Arnold, Inequalities: Theory of Majorization and Its Applications, Springer, New York, 2011. 
[14] B. J. McClelland, Properties of the latent roots of a matrix: The estimation of $\pi$ electron energies, J. Chem. Phys. 54 (1971) 640-643.

[15] V. Nikiforov, The energy of graphs and matrices, J. Math. Anal. Appl. 326 (2007) 1472-1475.

[16] S. Wagner, H. Wang, Introduction to Chemical Graph Theory, CRC Press, Boca Raton, 2019.

[17] A. Yu, M. Lu, F. Tian, New upper bounds for the energy of graphs, MATCH Commun. Math. Comput. Chem. 53 (2005) 441-448.

[18] X. Zhan, Inequalities for the singular values of Hadamard products, SIAM J. Matrix Anal. Appl. 18 (1997) 1093-1095.

[19] B. Zhou, Energy of graphs, MATCH Commun. Math. Comput. Chem. 51 (2004) 111-118. 